\title{
Pore Formation Coupled to Ion Transport through Lipid Membranes as Induced by Transmembrane Ionic Charge Imbalance: Atomistic Molecular Dynamics Study
}

\author{
Andrey A. Gurtovenko*,† and Ilpo Vattulainent,‡ \\ Laboratory of Physics and Helsinki Institute of Physics, Helsinki University of Technology, \\ P.O. Box 1100, FI-02015 HUT, Finland, and Memphys-Center for Biomembrane Physics, \\ Physics Department, University of Southern Denmark, Campusvej 55, DK-5230 Odense M, Denmark
}

Received May 13, 2005; E-mail: agu@fyslab.hut.fi; Ilpo.Vattulainen@csc.fi

Formation of transient water pores in biological membranes lies at the heart of many important cellular processes, including fusion events, maintenance of osmotic balance, drug and antibody delivery into cells, and ion transport across the membrane. Ion transport, in particular, is of central interest since membranes strive to maintain a cationic electrochemical gradient used for ATP synthesis as well as a variety of activities, such as transport of nutrients and conveyance of electrical signals. While maintaining the electrochemical gradient is essential, ions yet leak through lipid membranes. The mechanisms of ion leakage are not well understood, however.

In this Communication, we show that ion leakage can take place in protein-free membranes through transient water pores induced by a transmembrane ionic charge imbalance (see Figure 1). This mechanism can be expected in cells characterized by ionic concentration differences across the membrane. The view presented here further complements and lends support to previous studies ${ }^{1-4}$ for pore formation induced by external fields.

We have performed atomic-scale molecular dynamics (MD) simulations on dimyristoylphosphatidylcholine (DMPC) bilayers in the liquid crystalline phase ( $T=323 \mathrm{~K}$ ) using a united-atom forcefield of Berger et al. ${ }^{5}$ Recently, we have used that successfully in MD simulations for mixtures of charged lipids with DMPC. ${ }^{6}$ For completeness, we have also conducted simulations at the physiological temperature $(T=310 \mathrm{~K})$. To implement a transmembrane ionic charge imbalance, we employed a double bilayer setup (i.e., two distinct bilayers in a simulation box). ${ }^{7,8}$ All of the simulations were performed using the Gromacs suite. ${ }^{9}$ For further simulation details, see Supporting Information (SI).

The charge imbalance of six sodium ions per bilayer (i.e., the imbalance of $12 \mathrm{Na}^{+}$ions in the whole double bilayer system), imposed at the two sides of a DMPC bilayer of 128 lipids, was found to induce, on average, a transmembrane voltage of $\sim 2.85 \pm$ $0.49 \mathrm{~V}$ across the membrane (see Figure 2). This voltage corresponds to an electric field of $\sim 0.41 \mathrm{~V} / \mathrm{nm}$ (averaged over the simulation box), which is close to values used in previous studies to induce pore formation by an external electric field. ${ }^{1,2,4}$ The applied charge imbalance gives rise to a rapid formation of a water pore within $\sim 1 \mathrm{~ns}$ in one of the two bilayers, the other staying intact. The pore formation starts with the creation of a single water defect (a chain of water molecules) spanning the entire membrane in $\sim 0.5$ ns (see Figure 1B), which then expands rapidly within the next 0.5 ns (see Figures 1C and 3 as well as SI). The latter stage involves considerable redistribution of lipid headgroups close to the defect, surrounding and stabilizing the pore ${ }^{1,2,4}$ (see SI).

$\dagger$ Helsinki University of Technology.

¥University of Southern Denmark.
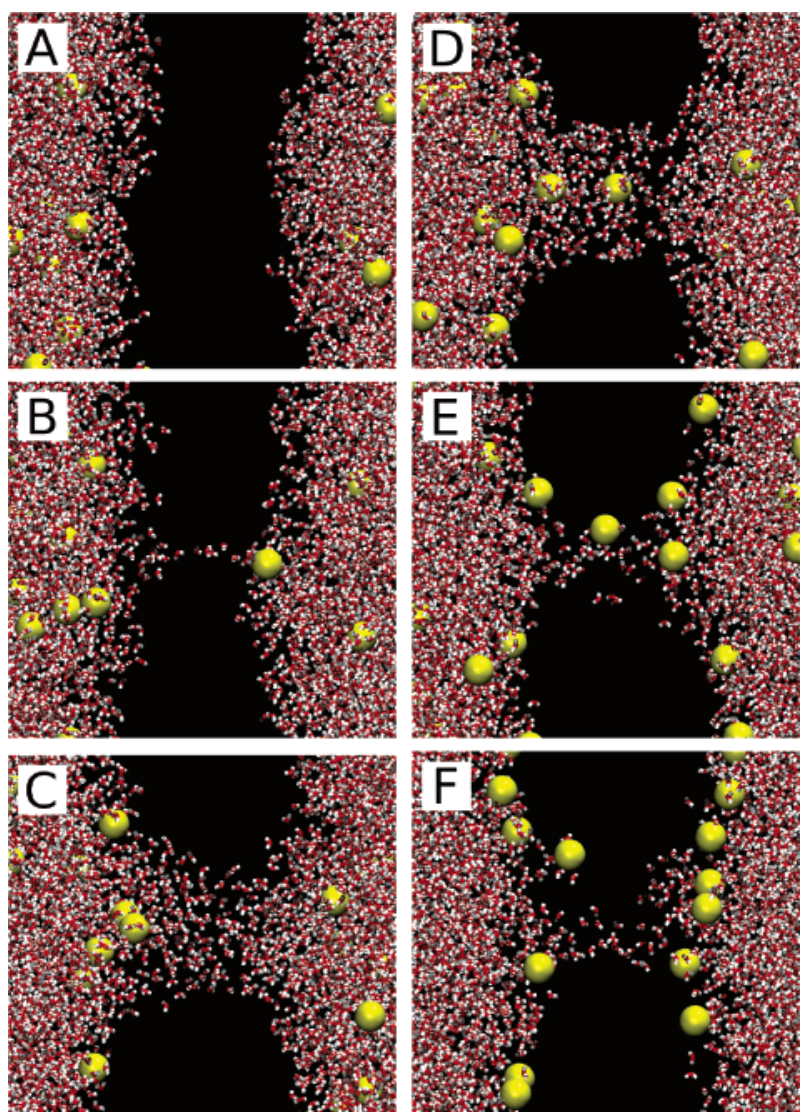

Figure 1. Pore formation coupled to ion transport induced by ion imbalance: (A) 20 ps, (B) 450 ps, (C) 1000 ps, (D) 1070 ps, (E) 9180 ps, and $(\mathrm{F}) 60 \mathrm{~ns}$. The membrane is not shown (black space); water is shown in red-white, and $\mathrm{Na}^{+}$ions are in yellow, with excess $\mathrm{Na}^{+}$on the lefthand side.

Once a pore has been formed, we find transport of $\mathrm{Na}^{+}$ions through the pore along the $\mathrm{Na}^{+}$concentration gradient (Figures 1D and 3). The permeation of $\mathrm{Na}^{+}$ions through the pore and the size of the pore are both controlled by the field: the higher the transmembrane electric field, the faster the motion of a $\mathrm{Na}^{+}$ion through the pore, and the larger the pore itself. First, two $\mathrm{Na}^{+}$ions permeate through the membrane very rapidly (within $0.5 \mathrm{~ns}$ ), reducing the charge imbalance and, correspondingly, discharging the transmembrane voltage down to $\sim 1.47 \mathrm{~V}$. This decreases significantly the number of water molecules inside the pore and slows down the ionic transport (see Figure 3). Consequently, the time required for permeation of the next (third) $\mathrm{Na}^{+}$ion increases by about an order of magnitude. The overall picture here is partly reminiscent of ion transport through a generic hydrophobic pore. ${ }^{10}$ 


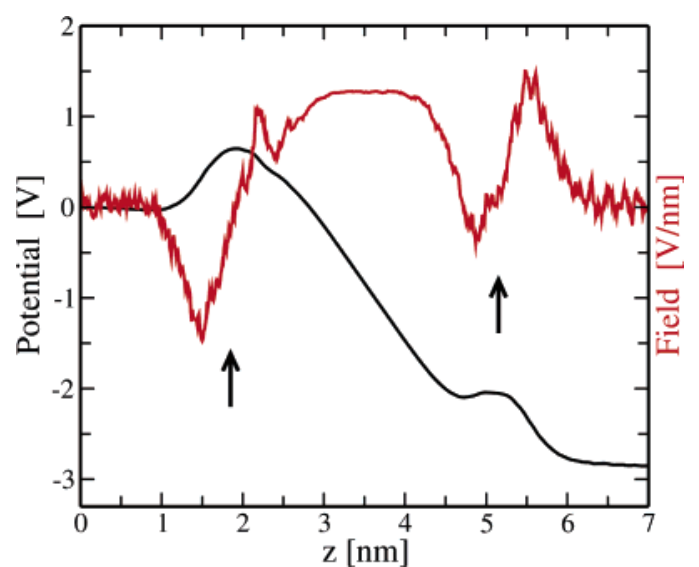

Figure 2. Electrostatic potential and electric field versus distance $z$ from the middle of the water phase between the two bilayers. The results correspond to a transmembrane charge imbalance of $6 \mathrm{Na}^{+}$ions per bilayer; average positions of phosphorus atoms of lipids for the two bilayer leaflets are shown by arrows.

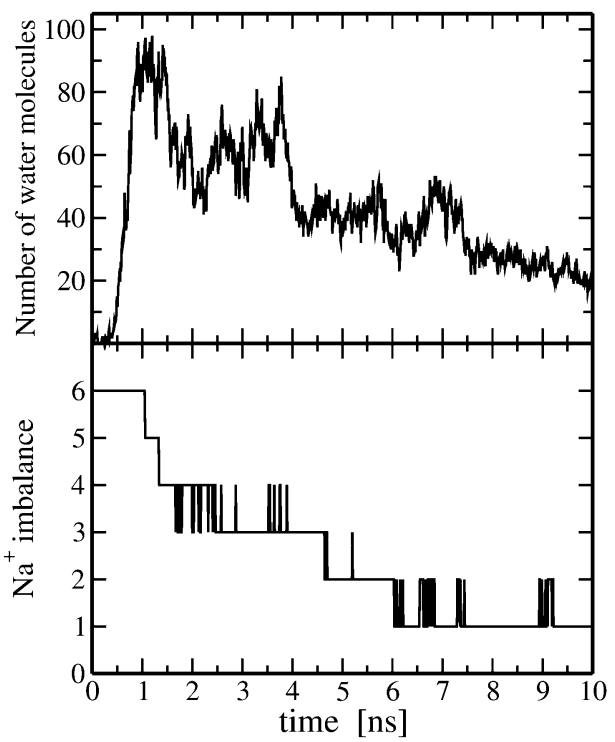

Figure 3. Time evolution for the number of water molecules in a pore within $0.5 \mathrm{~nm}$ from the bilayer center (top) and for the transmembrane $\mathrm{Na}^{+}$ ion imbalance (bottom).

MD simulations performed at a lower temperature $(T=310 \mathrm{~K})$ suggest that the general picture and the sequence of events remain the same, the only difference being the slowing down of pore formation and ionic transport roughly by a factor of 2-3 (see SI).

The decrease in ion mobility seems to be caused not only by a drop in the transmembrane voltage but also by interactions of sodium ions with lipids forming pore "walls" when the amount of water in the pore decreases significantly (see Figure 1E and SI). Because of this drop in ion mobility, the permeation of the last (sixth) $\mathrm{Na}^{+}$ion through the pore is not observed in the simulations: even after $60 \mathrm{~ns}$, there remains a charge imbalance of $1 \mathrm{Na}^{+}$ ion corresponding to a transmembrane voltage of $\sim 0.21 \mathrm{~V}$ (see SI).

The ion transport through a transient water pore was also observed at a smaller initial charge imbalance of $5 \mathrm{Na}^{+}$ions per bilayer, corresponding, on average, to a transmembrane voltage of $\sim 2.0 \pm 0.14 \mathrm{~V}$. Pore formation took then place after $5 \mathrm{~ns}$. Remarkably, the corresponding field of $\sim 0.29 \mathrm{~V} / \mathrm{nm}$ (averaged over the simulation box) is weaker than those found leading to pore formation in computer experiments which employed a constant electric field $(0.4 \mathrm{~V} / \mathrm{nm}$ has been reported as a minimal value for pore formation ${ }^{2}$ ). The low threshold value observed in our case is likely due to the stochastic nature of the electric field induced by an ionic concentration gradient: the field is mostly determined by instantaneous positions of $\mathrm{Na}^{+}$ions and, therefore, subject to considerable fluctuations in time and space with respect to its average value.

Interestingly, after leakage of 3 ions through a membrane for a system with an initial charge imbalance of $5 \mathrm{Na}^{+}$ions, we observed a closure of a water pore at $t \approx 58 \mathrm{~ns}$. In contrast, for a system with an initial charge imbalance of $6 \mathrm{Na}^{+}$ions, the water pore stayed open over the total time scale of $60 \mathrm{~ns}$ (see Figure 1F), while the residual charge imbalance $\left(1 \mathrm{Na}^{+}\right)$was 2 -fold smaller than that for the above system. This suggests that the small residual transmembrane potential does not play a crucial role in stabilizing a pore. Instead, after a drop of the transmembrane voltage below some critical value due to ion leakage, a water pore becomes metastable. To shed more light on this issue, we conducted a complementary simulation, where we removed the residual charge imbalance of 1 $\mathrm{Na}^{+}$ion from the system presented in Figure 3 at 50 ns (see SI). Having done that, the water pore turned out to be stable and insensitive to the voltage discharge for the first $6 \mathrm{~ns}$, but then it closed between 7 and $8 \mathrm{~ns}$. The closure seems to be associated with large fluctuations in the pore size rather than with a full discharge of the transmembrane potential.

Our results contribute to the long-standing discussion on how ions permeate protein-free lipid membranes, one of possible mechanisms being ion leakage through a transient water pore. ${ }^{11}$ Our MD study demonstrates a physically plausible mechanism of pore formation and the consequent ion transport due to large local fluctuations in ion concentrations on two sides of a lipid membrane. In living cells, density gradients of, for example, $\mathrm{H}^{+}$and $\mathrm{Na}^{+}$ions across a membrane are an inherent feature. That suggests this mechanism to be of generic nature. Understanding the mechanism allows one to design ways to better control processes related to water pores. Possibly, such a transmembrane charge imbalance can be also responsible for pore formation in a lipid membrane upon binding of cationic antibacterial proteins to the membrane surface, ${ }^{12}$ and it would be genuinely interesting to clarify the role of ionic imbalance on DNA translocation.

Acknowledgment. This work has been supported by the Academy of Finland through Grant Nos. 202598 (A.A.G.) and 80246 (I.V.), and by its Center of Excellence Program. The simulations were performed on the HorseShoe (DCSC) supercluster at the University of Southern Denmark.

Supporting Information Available: Simulation details and system setup, and further results. This material is available free of charge via the Internet at http://pubs.acs.org.

\section{References}

(1) Tieleman, D. P.; Leontiadou, H.; Mark, A. E.; Marrink, S.-J. J. Am. Chem. Soc. 2003, 125, 6382 .

(2) Tieleman, D. P. BMC Biochemistry 2004, 5, 10.

(3) Leontiadou, H.; Mark, A. E.; Marrink, S.-J. Biophys. J. 2004, 86, 2156.

(4) Tarek, M. Biophys. J. 2005, 88, 4045.

(5) Berger, O.; Edholm, O.; Jahnig, F. Biophys. J. 1997, 72, 2002.

(6) Gurtovenko, A. A.; Patra, M.; Karttunen, M.; Vattulainen, I. Biophys. J. 2004, 86, 3461 .

(7) Sachs, J. N.; Crozier, P. S.; Woolf, T. B. J. Chem. Phys. 2004, 121, 10847.

(8) Gurtovenko, A. A. J. Chem. Phys. 2005, 122, 244902.

(9) Lindahl, E.; Hess, B.; van der Spoel, D. J. Mol. Model. 2001, 7, 306.

(10) Dzubiella, J.; Allen, R. J.; Hansen, J.-P. J. Chem. Phys. 2004, 120, 5001.

(11) Paula, S.; Volkov, A. G.; VanHoek, A. N.; Haines, T. H.; Deamer, D. W. Biophys. J. 1996, 70, 339.

(12) Miteva, M.; Andersson, M.; Karshikoff, A.; Ottig, G. FEBS Lett. 1999, 462,155 .

JA053129N 